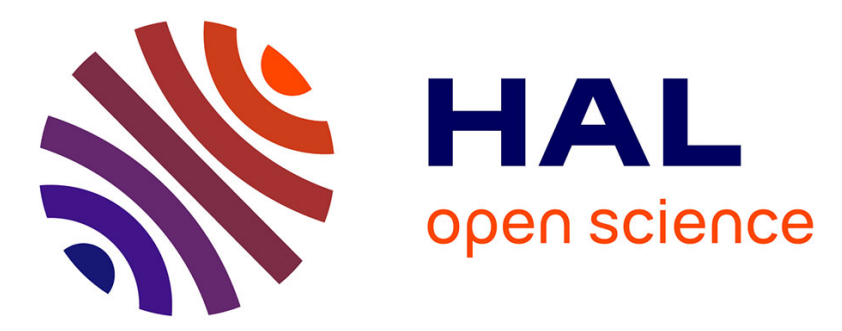

\title{
Basset-Boussinesq history force of a fluid sphere.
}

Dominique Legendre, Azeddine Rachih, Claire Souilliez, Sophie Charton, Éric

\author{
Climent
}

\section{To cite this version:}

Dominique Legendre, Azeddine Rachih, Claire Souilliez, Sophie Charton, Éric Climent. BassetBoussinesq history force of a fluid sphere.. Physical Review Fluids, 2019, 4 (7), pp.073603. 10.1103/PhysRevFluids.4.073603 . cea-02535280

\section{HAL Id: cea-02535280 https://hal-cea.archives-ouvertes.fr/cea-02535280}

Submitted on 9 Apr 2020

HAL is a multi-disciplinary open access archive for the deposit and dissemination of scientific research documents, whether they are published or not. The documents may come from teaching and research institutions in France or abroad, or from public or private research centers.
L'archive ouverte pluridisciplinaire HAL, est destinée au dépôt et à la diffusion de documents scientifiques de niveau recherche, publiés ou non, émanant des établissements d'enseignement et de recherche français ou étrangers, des laboratoires publics ou privés. 


\section{OATAO \\ Open Archive Toulouse Archive Ouverte}

\section{Open Archive Toulouse Archive Ouverte}

OATAO is an open access repository that collects the work of Toulouse researchers and makes it freely available over the web where possible

This is a publisher's version published in: http://oatao.univ-toulouse.fr/25644

Official URL:

https://doi.org/DOI:10.1103/PhysRevFluids.4.073603

\section{To cite this version:}

Legendre, Dominique and Rachih, Azeddine and Souilliez, Claire and Charton, Sophie and Climent, Éric BassetBoussinesq history force of a fluid sphere. (2019) Physical Review Fluids, 4 (7). 073603. ISSN 2469-990X.

Any correspondence concerning this service should be sent to the repository administrator: tech-oatao@listes-diff.inp-toulouse.fr 


\title{
Basset-Boussinesq history force of a fluid sphere
}

\author{
Dominique Legendre, ${ }^{1}$ Azeddine Rachih, ${ }^{1,2}$ Claire Souilliez, ${ }^{1}$ Sophie Charton, ${ }^{2}$ and Eric Climent ${ }^{1}$ \\ ${ }^{1}$ Institut de Mécanique des Fluides de Toulouse, Université de Toulouse, CNRS, INPT, UPS, 31400 \\ Toulouse, France \\ ${ }^{2}$ CEA, DEN, MAR, DMRC, SA2I, 30207 Bagnols-sur-Cèze, France
}

(Received 7 April 2019; published 26 July 2019)

\begin{abstract}
We consider the Basset-Boussinesq (history) force experienced by a spherical drop. We seek to determine the kernel of the Basset-Boussinesq force when internal circulation of the fluid occurs. We first characterize the slip at a fluid-sphere interface. Under both steady and unsteady conditions, the corresponding slip length is remarkably uniform along the fluid-sphere interface and is directly related to the viscosity ratio. Combining the analytical expression of the Basset-Boussinesq kernel obtained for a solid sphere with the interface slip and the obtained description of the slip at the fluid-fluid interface, we are able to describe the Basset-Boussinesq history force acting on a spherical drop. This expression is valid whatever the viscosity ratio from bubbles to viscous drops.
\end{abstract}

DOI: 10.1103/PhysRevFluids.4.073603

\section{INTRODUCTION}

Under the Stokes flow condition, the force experienced by a fluid sphere of radius $R$, viscosity $\mu_{i}$, and density $\rho_{i}$ moving with relative velocity $W(t)=U(t)-V(t)$ in an unbounded fluid of viscosity $\mu_{e}$ and density $\rho_{e}$ is composed of the steady drag force, the Basset-Boussinesq history force, and the added mass force [1,2]

$$
F(t)=6 \pi \mu_{e} R \frac{2+3 \mu^{*}}{3+3 \mu^{*}} W(t)+6 \pi \mu_{e} R \int_{0}^{t} \frac{d W}{d t^{\prime}} K_{\mu}\left(t-t^{\prime}, \mu^{*}\right) d t^{\prime}+\frac{2}{3} \rho_{e} \pi R^{3} \frac{d W}{d t},
$$

where $\mu^{*}=\mu_{i} / \mu_{e}$ is the viscosity ratio and $K_{\mu}$ is the memory kernel. The external Reynolds number $\operatorname{Re}=2 R W \rho_{e} / \mu_{e}$ should satisfy $\operatorname{Re} \ll 1$. A constant relative slip velocity will be denoted by $W_{0}$.

Here we obtain the general expression for $K_{\mu}$ as a function of $\mu^{*}$ for a fluid sphere. The analytic solution for the force has been obtained in the Fourier-transform space but the transform from the frequency domain to the time domain can only be achieved under the two limits of a solid sphere and spherical bubble [3,4]. The asymptotic limits for $K_{\mu}$ are discussed in [5].

In the solid sphere limit $\left(\mu^{*}=\infty\right)$, the Basset-Boussinesq history force is associated with the kernel $[6,7]$

$$
K_{\mu}\left(t, \mu^{*}=\infty\right)=\frac{1}{\sqrt{\pi t / t_{v}}}
$$

where $t_{v}=R^{2} / v_{e}$ is the characteristic diffusion timescale based on the kinematic viscosity $v_{e}=$ $\mu_{e} / \rho_{e}$ of the external fluid. We also introduce the characteristic diffusion time $t_{v i}=R^{2} / \nu_{i}$ based on the kinematic viscosity $v_{i}=\mu_{i} / \rho_{i}$ of the internal fluid. In contrast, in the bubble limit $\left(\mu^{*}=0\right)$, Yang and Leal [4] have demonstrated that

$$
K_{\mu}(t, \mu=0)=\frac{4}{3} \exp \left[9 t / t_{v}\right] \operatorname{erfc}\left[3 \sqrt{t / t_{v}}\right] .
$$


In parallel, the unsteady Stokes problem has also been considered for a slip sphere. The Navier slip condition is then applied to the sphere surface

$$
u_{\theta}=\lambda r \frac{\partial}{\partial r}\left(\frac{u_{\theta}}{r}\right) \text { for } r=R,
$$

where $u_{\theta}$ is the tangential velocity, $\lambda$ is the surface slip length, and $r$ is the radial position. The force experienced by the sphere is then $[3,8,9]$

$$
F(t)=6 \pi \mu_{e} R \frac{1+2 \lambda / R}{1+3 \lambda / R} W(t)+6 \pi \mu_{e} R \int_{0}^{t} \frac{d W}{d t^{\prime}} K_{\lambda}\left(t-t^{\prime}, \lambda\right) d t^{\prime}+\frac{2}{3} \pi \rho_{e} R^{3} \frac{d W}{d t},
$$

where the expression for the memory kernel $K_{\lambda}$ for a uniform slip along the surface is

$$
K_{\lambda}(t, \lambda)=\frac{(1+2 \lambda / R)^{2}}{\lambda / R(1+3 \lambda / R)} \exp \left[\frac{(1+3 \lambda / R)^{2}}{\lambda^{2} / R^{2}} t / t_{v}\right] \operatorname{erfc}\left[\frac{(1+3 \lambda / R)}{\lambda / R} \sqrt{t / t_{v}}\right] .
$$

The expression for $K_{\lambda}$ can be matched to the solution for a solid sphere and a spherical bubble considering asymptotic values of the slip length, i.e., $\lambda=0$ for a no-slip surface (solid sphere case) and $\lambda=\infty$ for a free-slip surface (bubble case), considering that $K_{\mu}\left(t, \mu^{*}=0\right)=K_{\lambda}(t, \lambda=\infty)$ and $K_{\mu}\left(t, \mu^{*}=\infty\right)=K_{\lambda}(t, \lambda=0)$.

The aim of this work is to first relate the slip length at a fluid-sphere interface to the viscosity ratio and then use it to extend the Basset-Boussinesq history force acting on a fluid sphere. The relevance of the proposed history force is validated by direct numerical simulations obtained with the JADIM code.

\section{NAVIER-STOKES SOLVER}

The computations in this paper were carried out with the JADIM code described in detail in previous studies. In particular, the JADIM code has been used for the simulation of bubble and particle dynamics [10-15]. The code has been extended for this study to handle simulations of unsteady flows around a viscous fluid sphere. With this aim, we solve numerically the incompressible Navier-Stokes equations for the velocity $\mathbf{u}_{\mathbf{k}}$ and the pressure $p_{k}$ for both the internal fluid $(k=i)$ and the external fluid $(k=e)$,

$$
\nabla \cdot \mathbf{u}_{\mathbf{k}}=0, \quad \rho_{k}\left(\frac{\partial \mathbf{u}_{\mathbf{k}}}{\partial t}+\mathbf{u}_{\mathbf{k}} \cdot \nabla \mathbf{u}_{\mathbf{k}}\right)=-\nabla p_{k}+2 \mu_{k} \boldsymbol{\nabla} \cdot \mathbf{S}_{\mathbf{k}},
$$

where $\mathbf{S}_{\mathbf{k}}=\left[\nabla \mathbf{u}_{\mathbf{k}}+\left(\nabla \mathbf{u}_{\mathbf{k}}\right)^{\top}\right] / 2$ is the rate-of-strain tensor. Associated with Eqs. (7) are the boundary conditions at the fluid-fluid interface, which read, for axisymmetric two-dimensional geometry,

$$
u_{e, r}=u_{i, r}=0, \quad u_{e, \theta}=u_{i, \theta}, \quad \mu_{e} e_{e, r \theta}=\mu_{i} e_{i, r \theta} .
$$

Numerically, the boundary conditions at the interface are implemented in the following manner. Using finite-difference discretization, the tangential strain rate on each side of the interface $S_{k, r \theta}=$ $\partial u_{k, \theta} / \partial r-u_{k, \theta} / R$ can be estimated with second-order accuracy using

$$
\begin{gathered}
\frac{\partial u_{e, \theta}}{\partial r}=-\frac{d_{e, 2}+d_{e, 3}}{d_{e, 2} d_{e, 3}} u_{e, \theta}+\frac{d_{e, 3}}{d_{e, 2}\left(d_{e, 3}-d_{e, 2}\right)} u_{e, \theta 2}-\frac{d_{e, 2}}{d_{e, 3}\left(d_{e, 3}-d_{e, 2}\right)} u_{e, \theta 3}, \\
\frac{\partial u_{i, \theta}}{\partial r}=\frac{d_{i, 2}-d_{i, 3}}{d_{i, 2} d_{i, 3}} u_{i, \theta}-\frac{d_{i, 3}}{d_{i, 2}\left(d_{i, 3}-d_{i, 2}\right)} u_{i, \theta 2}+\frac{d_{i, 2}}{d_{i, 3}\left(d_{i, 3}-d_{i, 2}\right)} u_{i, \theta 3},
\end{gathered}
$$

where $u_{k, \theta 2}$ and $u_{k, \theta 3}$ are the velocities placed at the center from the two first cells to the interface and located in fluid $k$ at distances $d_{k, 2}$ and $d_{k, 3}\left(d_{k, 2}<d_{k, 3}\right)$ from the interface, respectively. Combining Eqs. (8) and (9) for $k=i$ and $k=e$, the velocity at the interface is deduced from the calculated 
TABLE I. Drag coefficient for $\mu^{*}=1$ and $\rho^{*}=1$ at $\operatorname{Re}=0.1,0.5$, and 1 .

\begin{tabular}{lccccc}
\hline \hline Re & Present study & Relation (12) & Ref. [17] & Ref. [18] & Ref. [19] \\
\hline 0.1 & 205.3 & 203.1 & & 202.4 & \\
0.5 & 42.8 & 43.1 & 42.9 & 42.6 & 42.6 \\
1 & 22.4 & 23.1 & 22.4 & 22.5 & 22.4 \\
\hline \hline
\end{tabular}

velocity

$$
\begin{aligned}
u_{e, \theta}=u_{i, \theta}= & {\left[\frac{d_{e, 3}}{d_{e, 2}\left(d_{e, 3}-d_{e, 2}\right)} u_{e, \theta 2}-\frac{d_{e, 2}}{d_{e, 3}\left(d_{e, 3}-d_{e, 2}\right)} u_{e, \theta 3}+\mu^{*} \frac{d_{i, 3}}{d_{i, 2}\left(d_{i, 3}-d_{i, 2}\right)} u_{i, \theta 2}\right.} \\
& \left.-\mu^{*} \frac{d_{i, 2}}{d_{i, 3}\left(d_{i, 3}-d_{i, 2}\right)} u_{i, \theta 3}\right]\left[\frac{1}{R}+\frac{d_{e, 2}+d_{e, 3}}{d_{e, 2} d_{e, 3}}+\mu^{*}\left(\frac{d_{e, 2}+d_{e, 3}}{d_{e, 2} d_{e, 3}}-\frac{1}{R}\right)\right]^{-1} .
\end{aligned}
$$

Combining this expression with Eqs. (9) and (10), the calculation of the interfacial shear stress $\tau_{k, r \theta}=2 \mu S_{k, r \theta}$ involved in the local momentum balance ensures that the set of boundary conditions (8) is satisfied at the fluid-sphere interface.

The Navier-Stokes equations are solved with velocity-pressure variables in a general system of orthogonal curvilinear coordinates. The discretization is based on a staggered grid on which the equations are integrated in space using a finite-volume method with second-order accuracy. The advection and viscous terms are evaluated through second-order centered schemes whereas time advancement is achieved through a second-order time-accurate Runge-Kutta-Crank-Nicolson algorithm. Finally, incompressibility is satisfied at the end of each time step by solving a Poisson equation for an auxiliary potential. An axisymmetric mesh is made using a polar grid extending up to $R_{\infty}=50 R$ or $100 R$. The number of nodes is $N_{\theta}=34$ along the polar direction (from $\theta=0$ to $\theta=$ $\pi)$ and $N_{r}=20$ and $N_{r}=100$ along the radial direction inside and outside the drop, respectively. A specific condition needs to be implemented at $r=0$. Considering the staggered mesh used in JADIM, only radial velocities $u_{i, r}$ are located at nodes $r=0$. Using the symmetry of the problem, the value of $u_{i, r}$ for each node is interpolated using the mirror values located on the same radial direction.

A uniform distribution along the $\theta$ direction and a geometrical progression along the $r$ direction are used. The thickness $\delta$ of the closest cell to the drop surface is fixed to $\delta=0.01 R$ on both sides of the interface. The influence of these numerical parameters was carefully verified to make sure that the results are grid independent.

The developments of the code allowing for solving flows around fluid spheres have been validated considering a uniform steady flow of velocity $W=W_{0}$ for small to large Reynolds numbers Re and a wide range of viscosity ratio $\mu^{*}$. For the tests in the two limits $\mu^{*}=0$ (bubble) and $\mu^{*}=\infty$ (solid sphere), very good agreement was achieved with the values previously obtained with the same code JADIM $[13,14]$. We focus here on a series of validation performed for Reynolds number $\operatorname{Re} \leqslant 1$ and a density ratio $\rho^{*}=\rho_{i} / \rho_{e}=1$. For this range of Reynolds number the drag coefficient $C_{D}=2 F / \pi R^{2} \rho_{e} W_{0}^{2}$ is supposed to follow the Stokes solution corrected by the Oseen term [16]

$$
C_{D}=\frac{24}{\operatorname{Re}} \frac{2+3 \mu^{*}}{3+3 \mu^{*}}+\frac{9}{2}\left(\frac{2+3 \mu^{*}}{3+3 \mu^{*}}\right)^{2} .
$$

Our simulations are compared to the prediction of the relation (12) in Table I for $\mu^{*}=1$ at $\operatorname{Re}=0.1$, 0.5 , and 1. Numerical values obtained by Rivkind et al. [17], Oliver and Chung [18], and Feng and Michaelides [19] are also reported. Very good agreement is observed with previous works.

The normalized drag coefficient defined as $C_{D}^{*}=\left[C_{D}\left(\operatorname{Re}, \mu^{*}\right)-C_{D}(\operatorname{Re}, 0)\right] /\left[C_{D}(\operatorname{Re}, \infty)-\right.$ $\left.C_{D}(\mathrm{Re}, 0)\right]$ is plotted as a function of $\mu^{*}$ in Fig. 1 for different Reynolds numbers. Considering 


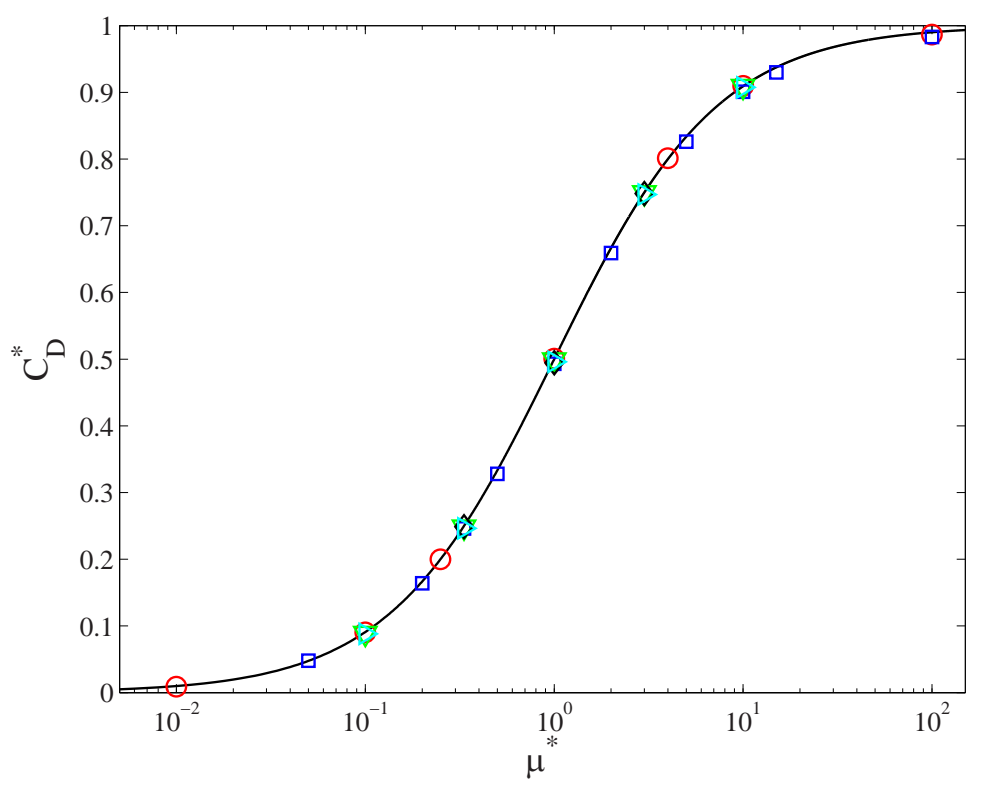

FIG. 1. Evolution of the normalized drag coefficient $C_{D}^{*}$ with the viscosity ratio $\mu^{*}$. The following denotations are used for numerical simulations: $\circ, \operatorname{Re}=0.1 ; \square, \operatorname{Re}=1 ;-$, relation (13); and from Ref. [18], $\nabla, \operatorname{Re}=0.1 ; *, \operatorname{Re}=0.5 ;$ and $\diamond, \operatorname{Re}=1$.

the analytical solution (12), $C_{D}^{*}$ is expected to evolve as

$$
C_{D}^{*}=\frac{\mu^{*}}{1+\mu^{*}}+O(\operatorname{Re})
$$

in the limit $\operatorname{Re} \rightarrow 0$, which is accurately reproduced by our simulations for $\operatorname{Re}=0.1$ and also $\operatorname{Re}=1$. The numerical results of Oliver and Chung [18] for $\operatorname{Re}=0.1,0.5$, and 1 also confirm that the relation (13) predicts correctly the dependence of the normalized drag coefficient $C_{D}^{*}$ with $\mu^{*}$ up to $\operatorname{Re}=1$.

\section{RELATION BETWEEN SLIP LENGTH AND VISCOSITY RATIO AT A FLUID-SPHERE INTERFACE}

\section{A. Steady uniform flow}

We consider a steady uniform flow of velocity $W_{0}$ around a fixed fluid sphere of radius $R$. The stream functions for the flow inside and outside the fluid sphere are, respectively [20,21],

$$
\begin{gathered}
\Psi_{i}=-\frac{W_{0} r^{2}}{4} \frac{1}{1+\mu^{*}}\left[1-\frac{r^{2}}{R^{2}}\right] \sin ^{2} \theta, \\
\Psi_{e}=\frac{W_{0} r^{2}}{2}\left[1-\frac{2+3 \mu^{*}}{1+\mu^{*}} \frac{R}{2 r}+\frac{\mu^{*}}{1+\mu^{*}} \frac{R^{3}}{2 r^{3}}\right] \sin ^{2} \theta .
\end{gathered}
$$

From this solution we can calculate the tangential velocity

$$
u_{e, \theta}=\frac{1}{2} \frac{1}{1+\mu^{*}} W_{0} \sin \theta
$$




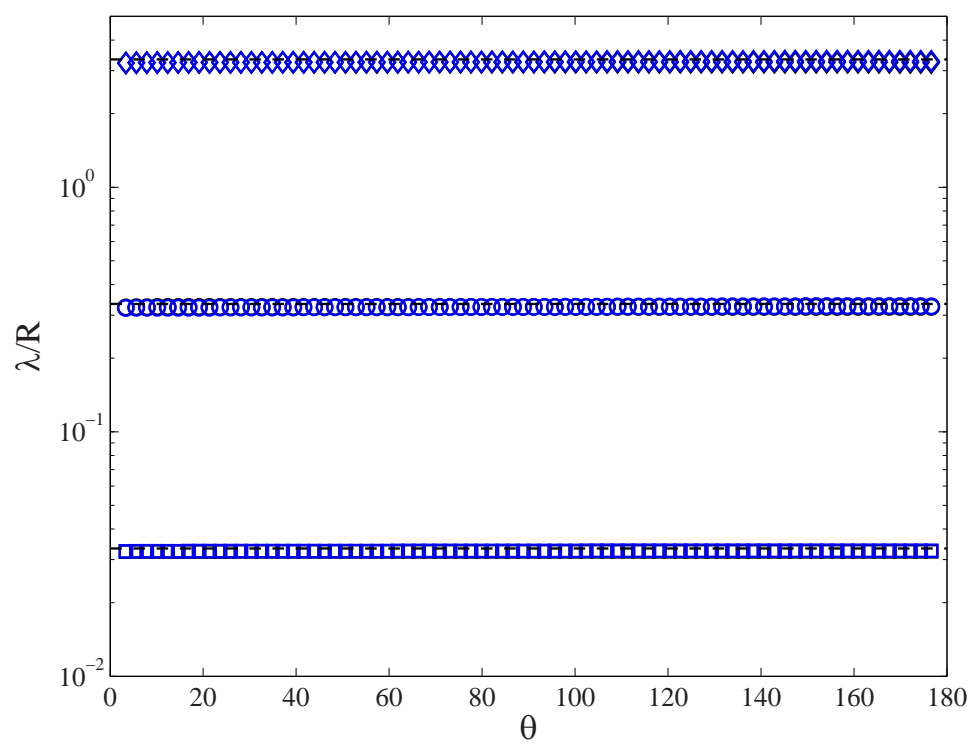

FIG. 2. Effective interface slip length $\lambda$ for a steady uniform flow obtained from numerical simulations. The interface distribution of $\lambda$ is shown as a function of angular position $\theta$ for $\operatorname{Re}=0.01$ (black symbols) and $\operatorname{Re}=0.1$ (blue symbols) for $\mu^{*}=0.1(\diamond), \mu^{*}=1(\circ)$, and $\mu^{*}=10(\square)$. The dashed line denotes the expression (18).

and the velocity gradient

$$
r \frac{\partial}{\partial r}\left(\frac{u_{e, \theta}}{r}\right)=\frac{3}{2} \frac{\mu^{*}}{1+\mu^{*}} W_{0} \sin \theta
$$

at the fluid sphere interface $(r=R)$. Considering the Navier-slip boundary condition at the interface given by Eq. (4), we can define an effective surface slip length $\lambda$ for the external fluid. From Eqs. (16) and (17) we show that for the steady Stokes flow the slip length is uniform at the interface and is directly related to the viscosity ratio by the relation

$$
\lambda=\frac{R}{3 \mu^{*}} .
$$

This expression is valid in the limit $\operatorname{Re} \rightarrow 0$. Note that in [22] a drop with an imposed slip at the interface is considered while the slip given by (18) results from the "natural" boundary conditions at a clean drop interface. Typical interface distributions of $\lambda$ obtained from the Navier-Stokes solver JADIM are reported in Fig. 2. Here $\lambda / R$ is reported as a function of the angular position $\theta$ for $\operatorname{Re}=$ 0.01 and 0.1 . Different values of $\mu^{*}$ are considered: $\mu^{*}=0.1,1$, and 10 . As shown, $\lambda$ is remarkably uniform and close to the value $R / 3 \mu^{*}$. The maximum deviation of $\lambda$ is less than $2 \%$ for all the simulated cases.

The evolution of the mean interfacial value of $\lambda$ with the viscosity ratio $\mu^{*}$ is reported in Fig. 3 . Different Reynolds numbers were considered $(\operatorname{Re}=0.01,0.1$, and 1$)$. As evidenced by the figure, all the simulations collapse onto the relation (18), while no noticeable effect of the Reynolds number on $\lambda$ is observed for the range of Reynolds number considered.

It should also be noted that we have compared the meshes of spatial extents $50 R$ and $100 R$ (not shown here for clarity). Both meshes give very close values for the interface slip length. 


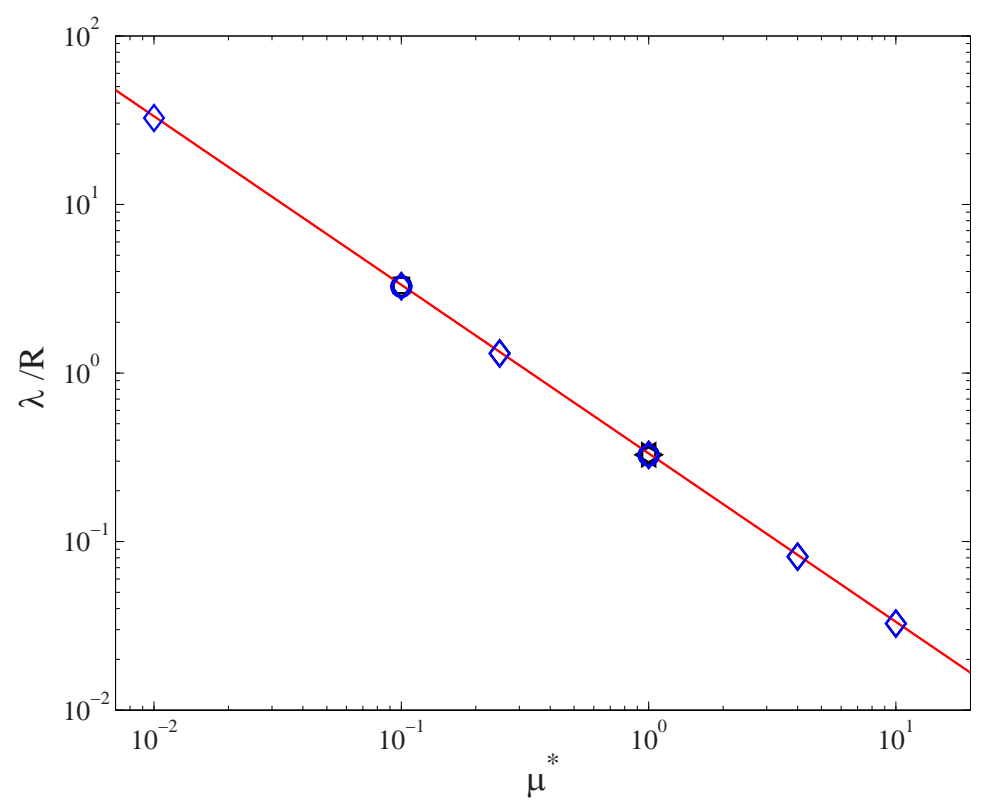

FIG. 3. Effective interface slip length $\lambda$ for a steady uniform flow as a function of $\mu^{*}$ for $\operatorname{Re}=0.01$ (o), $\operatorname{Re}=0.1(\diamond)$, and $\operatorname{Re}=1(\square)$. The solid line denotes $\lambda=R / 3 \mu^{*}$ [Eq. (18)].

\section{B. Unsteady uniform flow}

We now analyze the variation of the interfacial slip length $\lambda$ under unsteady conditions. For that purpose, the case of a fixed fluid sphere suddenly immersed in a uniform flow of velocity

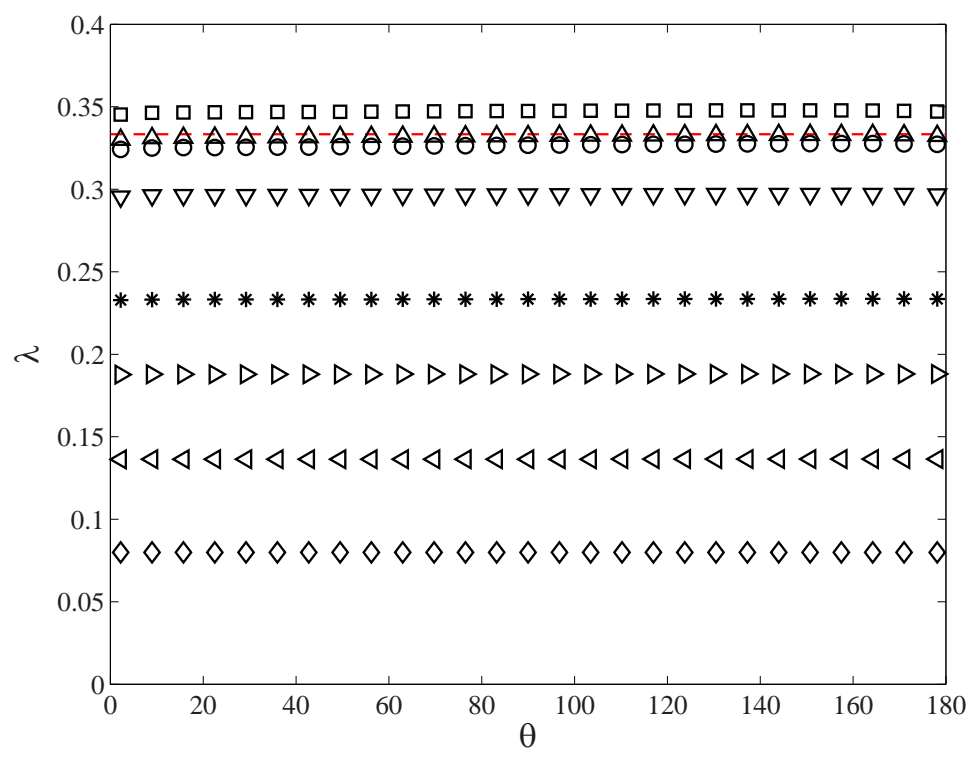

FIG. 4. Effective interface slip length $\lambda$ as a function of the angular position $\theta$ for $\operatorname{Re}=0.1$ and $\mu^{*}=1$ at different times: $t / t_{v}=0.002(\diamond), t / t_{v}=0.006(\triangleleft), t / t_{v}=0.012(\triangleright), t / t_{v}=0.02(*), t / t_{v}=0.04(\nabla), t / t_{v}=$ $0.12(\square), t / t_{v}=0.4(\triangle), t / t_{v}=1(\circ)$. The dashed line denotes $\lambda_{E}=R / 3 \mu^{*}$ [relation (18)]. 


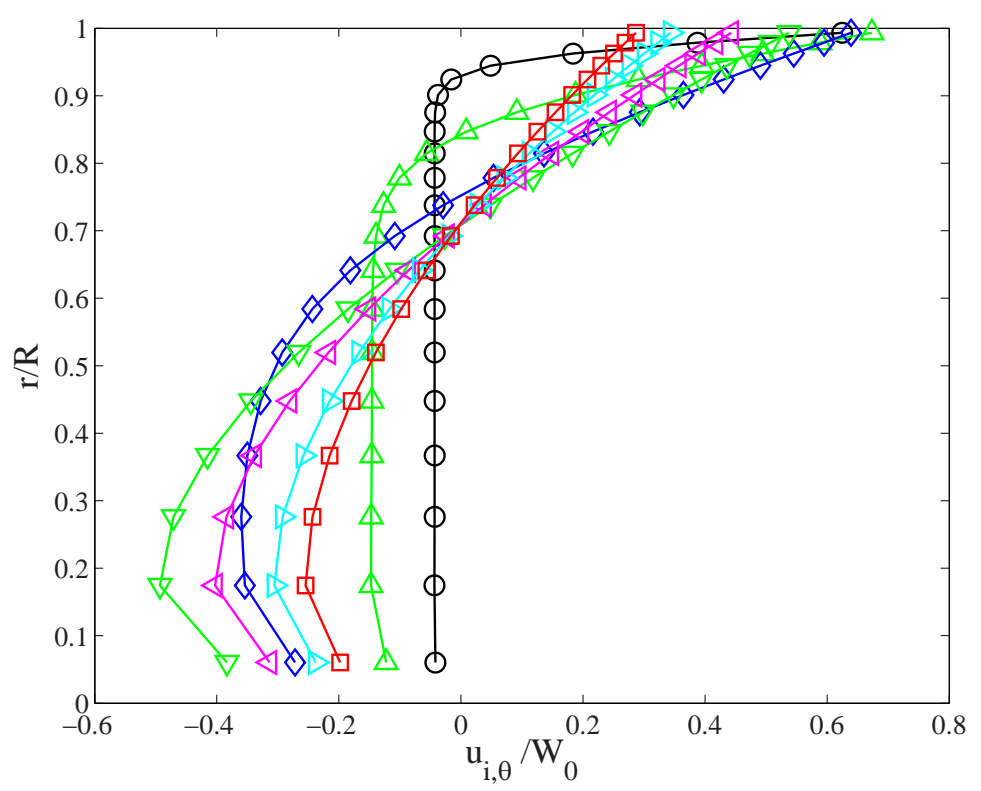

FIG. 5. Tangential velocity profile across the middle of the fluid sphere $\left(\theta=90^{\circ}\right)$ for $\operatorname{Re}=0.1$ and $\mu^{*}=1$ at different times: $t / t_{v}=0.0002(\circ), t / t_{v}=0.002(\triangle), t / t_{v}=0.01(\diamond), t / t_{v}=0.04(\nabla), t / t_{v}=0.1(\triangleleft), t / t_{v}=$ $0.4(\triangleright)$, and $t / t_{v}=\infty$ (steady state) $(\square)$.

$W_{0}$ is considered. Distributions of $\lambda$ along the interface at $\operatorname{Re}=0.1$ and $\mu^{*}=1$ are reported in Fig. 4 at different times. As shown, $\lambda$ starts from $\lambda=0$ and then increases in time. As observed for the steady uniform flow, the slip is remarkably uniform along the interface for all the times considered. A maximum is reached around $t=0.12 t_{\nu}$. Then $\lambda$ tends to $R / 3 \mu^{*}$ as expected for steady state. It should be stressed here that this asymptotic value is reached for a time $1 / 10$ of the characteristic diffusion time $t_{v}$. The evolution of $\lambda$ is directly connected to the development of the internal recirculation as illustrated in Fig. 5 for $\operatorname{Re}=0.1$ and $\mu^{*}=1$. The liquid is first shear driven close to the interface, generating the internal recirculation, and then a maximum reversal velocity near the drop axis of symmetry is observed, corresponding to the maximum slip value observed in Fig. 4. Then, due to momentum diffusion across the fluid sphere, both the interfacial velocity and the reversal velocity decrease and converge to their steady-state values.

The time evolution of $\lambda$ is reported in Fig. 6 for $\mathrm{Re}=0.1$ and different viscosity ratios. Two time scalings are presented: $t / t_{v}$ [Fig. 6(a)] and $t / t_{v i}$ [Fig. 6(b)]. As shown, all the time evolutions follow a unique curve when $\lambda \mu^{*}$ is reported as a function of $t / t_{v i}$, thus highlighting that $t_{v i}$ is the relevant time to describe the time evolution of the interfacial slip of a fluid sphere. The interface slip length $\lambda$ increases, reaching a maximum slightly larger than $R / 3 \mu^{*}$, and then rapidly decreases toward the plateau value $\lambda=R / 3 \mu^{*}$ for $t>0.15 t_{v i}$. The maximum value $\lambda_{\max }$ reached by the slip decreases when the viscosity ratio is increased for a given Re. The $\lambda_{\max }$ can be roughly fitted by $\lambda_{\max }=$ $\left[1+0.0047\left(\mu^{*}\right)^{1 / 3}\right] R / 3 \mu^{*}$, while the transient evolution for the slip length can be approximated by the equation

$$
\lambda=\frac{R}{3 \mu^{*}}\left\{1-\exp \left[-\left(60 t / t_{v i}\right)^{0.55}\right]\right\} \cos \left(20 t / t_{v i}\right),
$$

where the coefficients have been adjusted to the evolution observed for $\mu^{*}=1$. This relation, reported in Fig. 6(b) using a solid blue line, clearly describes the fast increase of the slip and the relaxation time necessary to reach the steady value. 

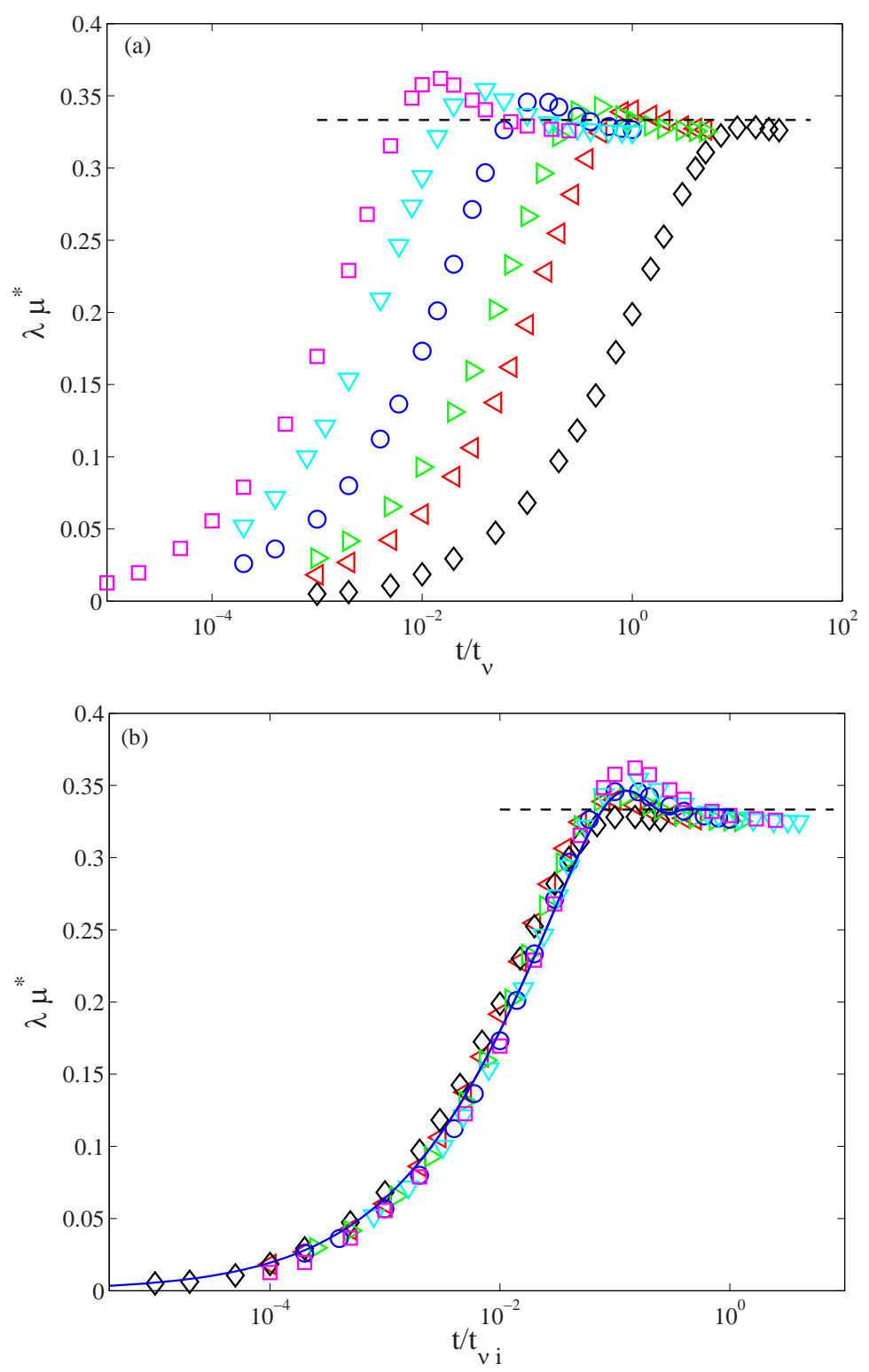

FIG. 6. Time evolution of the effective interface slip length $\lambda$ for $\operatorname{Re}=0.1$. Here $\lambda \mu^{*}$ is plotted as a function of (a) $t / t_{v}$ and (b) $t / t_{v i}$ for $\mu^{*}=0.01(\diamond), \mu^{*}=0.1(\triangleleft), \mu^{*}=0.25(\triangleright), \mu^{*}=1.0(\bigcirc), \mu^{*}=4.0$ $(\nabla), \mu^{*}=10.0(\square)$. The dashed line denotes $\lambda_{E}=R / 3 \mu^{*}$ [relation (18)] and the solid line relation (19).

\section{MEMORY KERNEL $K_{\mu}$ OF A FLUID SPHERE}

Replacing $\lambda$ by $R / 3 \mu^{*}$ in the steady drag force [first term in Eq. (5)] enables us to recover the dependence of the drag force on the viscosity ratio [first term in Eq. (1)]. The idea is now to apply the same substitution to the memory kernel. Due to the numerical simulations, we have highlighted that the slip length is uniform along a fluid-fluid interface for the range of small Reynolds number considered here. We first consider the memory kernel obtained by simply combining the relation 


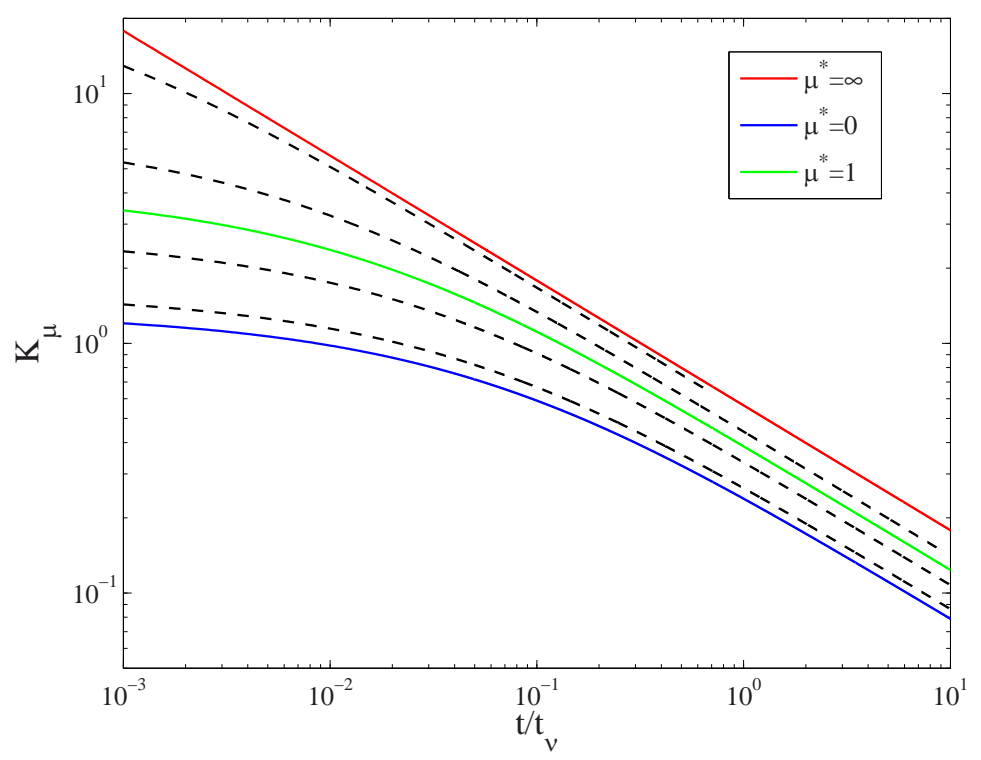

FIG. 7. Evolution of the memory kernel for different viscosity ratios. From top to bottom the solid lines denote $\mu^{*}=\infty$ (red curve), $\mu^{*}=1$ (green curve), and $\mu^{*}=0$ (blue curve) and the dashed lines stand for $\mu^{*}=10,2,0.5,01$.

(18) and the kernel expression (6):

$$
K_{\mu}\left(t, \mu^{*}\right)=\frac{\left(2+3 \mu^{*}\right)^{2}}{3\left(1+\mu^{*}\right)} \exp \left[9\left(1+\mu^{*}\right)^{2} t / t_{\nu}\right] \operatorname{erfc}\left[3\left(1+\mu^{*}\right) \sqrt{t / t_{\nu}}\right] .
$$

This expression is actually equivalent to expressions (2) and (3) in the limits $\mu^{*}=\infty$ (solid sphere) and $\mu^{*}=0$ (spherical bubble), respectively. The evolution of $K_{\mu}$ given by the relation (20) for different values of $\mu^{*}$ is reported in Fig. 7. Varying $\mu^{*}$ allows the evolution of $K_{\mu}$ from the spherical particle to the spherical bubble limit. The case $\mu^{*}=1$ lies in between these two limits.

The force's expression given by Eq. (1) is now compared to the force obtained by direct numerical simulations with the JADIM code for different viscosity ratios $\mu^{*}$. For a fluid sphere suddenly submitted to a uniform relative flow $W_{0}$ the experienced force is

$$
F(t)=6 \pi \mu_{e} R \frac{2+3 \mu^{*}}{3+3 \mu^{*}} W_{0}+6 \pi \mu_{e} R K_{\mu}(t) W_{0}
$$

Thus, subtracting the steady drag force from the unsteady force $F(t)$ gives direct access to the memory Kernel $K_{\mu}(t)$. The time evolution of $K_{\mu}(t)$ obtained from direct numerical simulation is reported in Fig. 8 for $\mathrm{Re}=0.1$. It is compared to the relation (20), reported using a dashed line. The proposed relation is observed to correctly describe the memory kernel for a large range of time.

Regarding the long time behavior, the effect of the Oseen wake is known to become more effective than the viscous diffusion $[23,24]$, so the sphere adjusts more rapidly to the velocity changes than predicted by the Basset-Boussinesq memory force. Thus, for time larger than $v / W_{0}^{2}$, i.e., $t>t_{v} \mathrm{Re}^{-2}$, the kernel decays faster as observed in Fig. 8 .

Considering now the behavior at early time, the kernel's evolution correctly fits with the relation (20) (reported using a dash-dotted line) once the slip has reached the value $\lambda=R / 3 \mu^{*}$, evidencing that for times larger than $0.15 t_{v i}$, the evolution of the kernel is accurately described using the relation (20). For early times, i.e., smaller than $0.15 t_{v i}$, the unsteady behavior of the interface slip needs to be considered. For that purpose, we propose to combine the fit given by the relation (19) obtained 


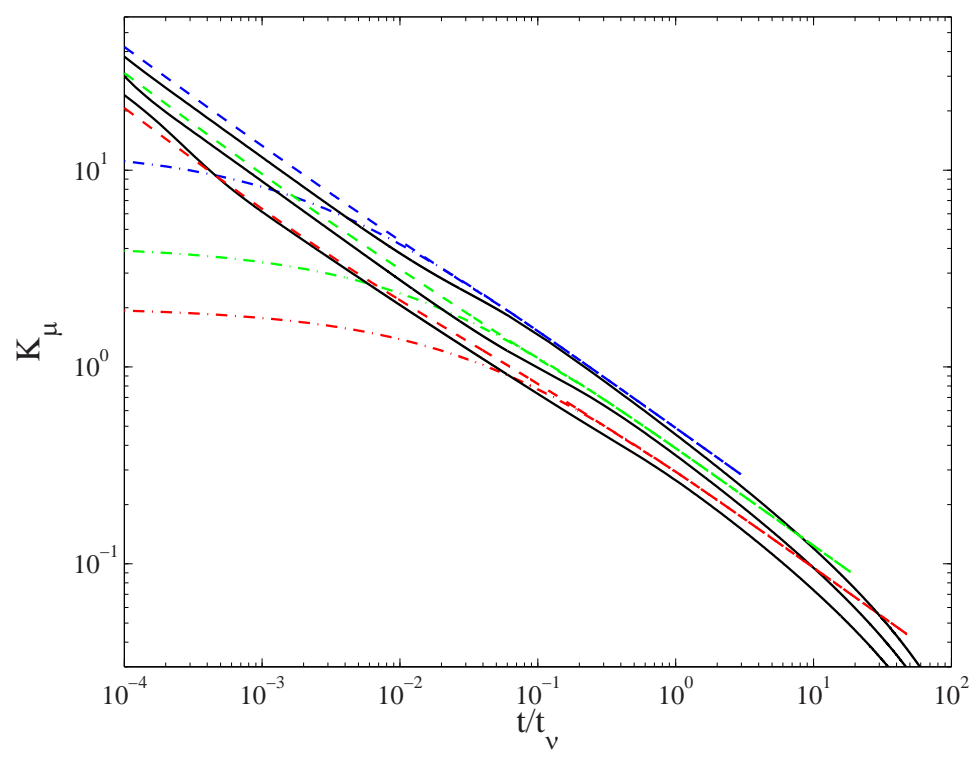

FIG. 8. Evolution of the memory kernel $K_{\mu}(t)$ (solid black lines). Also shown are values measured in the numerical simulations for different viscosity ratio $\mu^{*}$ at $\operatorname{Re}=0.1: \mu^{*}=0.25$ (red), $\mu^{*}=1$ (green), and $\mu^{*}=4$ (blue). Dash-dotted lines represent the relation (20) and dashed lines the kernel expression (6) combined with the unsteady slip length $\lambda(t)$ given by Eq. (19).

for $\lambda(t)$ with the kernel expression (6). The corresponding evolution is reported in Fig. 8 using solid lines. As shown, the early time evolution is now correctly described by the proposed memory kernel.

Finally, our simulations indicate that the general force equation (1) combined with the kernel expression (20) can be used to model unsteady fluid-sphere motion as long as the characteristic timescale of the flow variation is larger than $0.15 t_{v i}$. For higher frequency, adapted time evolutions of the interface slip length need to be provided. The corresponding expression (19) is suitable for a fluid sphere suddenly submitted to a uniform flow.

\section{CONCLUSION}

We have considered in this study the Basset-Boussinesq (history) force experienced by a fluid sphere. First, the slip at a fluid-sphere interface was considered. We showed that for both steady and unsteady conditions, the slip length distribution is remarkably uniform along the fluid-sphere interface and is directly related to the viscosity ratio. Combining the analytical expression of the Basset-Boussinesq kernel for a slip sphere and the description of the slip length at the interface of a fluid particle, we were able to describe the Basset-Boussinesq history force acting on a fluid sphere whatever the viscosity ratio considered, i.e., for spherical bubbles, drops, and particles.

\section{ACKNOWLEDGMENTS}

This work was financially supported by CEA's Nuclear Energy Division (SIACY Program). Calculations were run at the computational center CALMIP (Project No. P18028). We would like to thank Annaïg Pedrono for help and support with JADIM. 
[1] R. Gatignol, The Faxen formulas for a rigid particle in an unsteady non-uniform Stokes flow, J. Mec. Theor. Appl. 1, 143 (1983).

[2] M. R. Maxey and J. J. Riley, Equation of motion for a small rigid sphere in a nonuniform flow, Phys. Fluids 26, 883 (1983).

[3] E. E. Michaelides and Z.-G. Feng, The equation of motion of a small viscous sphere in an unsteady flow with interface slip, Int. J. Multiphase Flow 21, 315 (1995).

[4] S.-M. Yang and L. G. Leal, A note on memory-integral contributions to the force on an accelerating spherical drop at low Reynolds number, Phys. Fluids 3, 1822 (1991).

[5] V. Galindo and G. Gerbeth, A note on the force on an accelerating spherical drop at low-Reynolds number, Phys. Fluids 5, 3290 (1993).

[6] A. B. Basset, On the motion of a sphere in a viscous liquid, Philos. Trans. R. Soc. 179, 43 (1888).

[7] J. Boussinesq, Sur la résistance qu'oppose un liquide indéfini en repos, C. R. Acad. Sci. 100, 935 (1885).

[8] R. Gatignol, On the history term of Boussinesq-Basset when the viscous fluid slips on the particle, C. R. Mec. 335, 606 (2007).

[9] A. R. Premlata and H.-H. Wei, The basset problem with dynamic slip: Slip-induced memory effect and slip-stick transition, J. Fluid Mech. 866, 441 (2019).

[10] R. Adoua, D. Legendre, and J. Magnaudet, Reversal of the lift force on an oblate bubble in a weakly viscous linear shear flow, J. Fluid Mech. 628, 23 (2009).

[11] Y. Hallez and D. Legendre, Interaction between two spherical bubbles rising in a viscous liquid, J. Fluid Mech. 673, 406 (2011).

[12] D. Legendre, On the relation between the drag and the vorticity produced on a clean bubble, Phys. Fluids 19, 018102 (2007).

[13] D. Legendre and J. Magnaudet, The lift force on a spherical body in a viscous linear shear flow, J. Fluid Mech. 368, 81 (1998).

[14] J. Magnaudet, M. Rivero, and J. Fabre, Accelerated flows past a rigid sphere or a spherical bubble. Part 1. Steady straining flow, J. Fluid Mech. 284, 97 (1995).

[15] A. Merle, D. Legendre, and J. Magnaudet, Forces on a high-Re spherical bubble in a turbulent flow, J. Fluid Mech. 532, 53 (2005).

[16] T. Taylor and A. Acrivos, On the deformation and drag of a falling viscous drop at low Reynolds number, J. Fluid Mech. 18, 466 (1964).

[17] V. Y. Rivkind, G. M. Ryskin, and G. A. Fishbein, Flow around a spherical drop in a fluid medium at intermediate Reynolds numbers, Appl. Math. Mech. 40, 687 (1976).

[18] D. L. R. Oliver and J. N. Chung, Steady flows inside and around a fluid sphere at low Reynolds numbers, J. Fluid Mech. 154, 215 (1985).

[19] Z. Feng and E. Michaelides, The drag coefficients of a viscous sphere at intermediate and high Reynolds numbers, J. Fluids Eng. 123, 841 (2001).

[20] J. S. Hadamard, Mouvement permanent lent d'une sphère liquide et visqueuse dans un liquide visqueux, C. R. Acad. Sci. Paris 152, 1735 (1911).

[21] W. Rybczynski, On the translatory motion of a fluid sphere in a viscous medium, Bull. Acad. Sci. Cracow., Series A, 40 (1911).

[22] Z.-G. Feng, E. E. Michaelides, and S. Mao, On the drag force of a viscous sphere with interfacial slip at small but finite Reynolds numbers, Fluid Dyn. Res. 44, 025502 (2012).

[23] P. M. Lovalenti and J. F. Brady, The force on sphere in a uniform flow with small-amplitude oscillations at finite Reynolds number, J. Fluid Mech. 256, 607 (1993).

[24] R. Mei and R. J. Adrian, Flow past a sphere with an oscillation in the free-stream and unsteady drag at finite Reynolds number, J. Fluid Mech. 237, 133 (1992). 